\title{
THE THEORY OF MULTIPLE IMPLICATION AND ITS APPLICATION TO THE GENER- ALIZED PROBLEM OF EPIMENIDES
}

\author{
BY H. B. SMITH
}

Let us allow $p, q, r, \cdots$ to stand for propositional functions, that is, for variables whose truth-values are in general dependent on the meaning of the terms that enter into them, and let the expression $(p q r \ldots)$ have the following verbal interpretation:

" $p, q, r, \cdots$ are simultaneously true for some meanings of the terms that enter into them."

We shall speak of this expression, $(p q r \cdots)$, or its negative, as the existential of the $n$ elements, $p, q, r, \cdots$, and we shall say that it is of the first order and $n$th degree. The function containing existentials of the first order or their negatives as elements, will be of the second order and so on. For the particular case of one variable we should write:

$(p)=p$ is true for some meanings of the terms,

$(p)^{\prime}=p$ is true for no meanings of the terms,

$\left(p^{\prime}\right)^{\prime}=p$ is true for all meanings of the terms,

$\left(p^{\prime}\right)=p$ is true for not all meanings of the terms, the prime being used as the symbol of denial or negation.

The effect of the symbol ( ), regarded as an operator, will be in general to weaken the expression on which it operates, unless this expression be zero $(o)$ or one $(i)$. Thus,

$$
p \text { implies }(p) \text {, }
$$

$\left(p^{\prime}\right)^{\prime}$ implies $p$,

but not conversely. This simple provision is important, for it will lead at once to the result that certain generally recognized principles are untrue in a logic of complete generality. Thus it will turn out that the equality,

$$
p \text { implies } q=p^{\prime} \text { or } q
$$


does not hold true without limitation, namely, the limitation that the variables stand only for "propositions," for zero or one values, and not for propositional functions. This limitation is one that reduces the generality of our science and is directly related to an application of our theory which we shall make before we end. If we write,

$$
p<q=p \text { implies } q \text {, }
$$

we shall be prepared to set down the following definition,

$$
(p q)^{\prime}=p<q^{\prime}
$$

and from this follows the law of expansion for the general case,

$$
(p q r \cdots)^{\prime}=p q<r^{\prime}+\cdots=p<q^{\prime}+r^{\prime}+\cdots,
$$

that is, the elements are conceived to be conjoined as in a product, so that the consequent becomes the sum of the contradictories of the separate parts.

We might, if we liked, derive the fundamental laws of the calculus of propositions by assuming certain very simple properties of the existential, together with the fundamental equations,

$$
\begin{aligned}
(p q) & =p q+p^{\prime} q^{\prime}(p)(q)+(p)\left(q^{\prime}\right)^{\prime}+(q)\left(p^{\prime}\right)^{\prime}, \\
(p q)^{\prime} & =(p)^{\prime}+(q)^{\prime}+p^{\prime} q\left(q^{\prime}\right)+q^{\prime} p\left(p^{\prime}\right), \\
(\overline{p q}) & =\left(p^{\prime}\right)+\left(q^{\prime}\right), \\
(\overline{p q})^{\prime} & =\left(p^{\prime}\right)^{\prime}\left(q^{\prime}\right)^{\prime},
\end{aligned}
$$

the bar over $p q$ standing for the negation of the product, or $p^{\prime}+q^{\prime}$. Or, we can derive the simple properties of the existential from the laws of the calculus of propositions. Obviously we may raise the degree of the function without changing its value, by adding to it as many one-elements as we choose. This fact is important, because it will enable us to provide that the symbol of implication can always be made to appear in our development. Thus $\left(o^{\prime}=i\right)$,

$$
\begin{aligned}
& (p)^{\prime}=(i p)^{\prime}=i<p^{\prime}, \\
& (p)^{\prime}=(p i)^{\prime}=p<0 .
\end{aligned}
$$

And it is clear, too, that the elements may be permuted in any order, for 


$$
(p q)^{\prime}=p<q^{\prime}=q<p^{\prime}=(q p)^{\prime},
$$

and that no change will result if we add to the existential as many elements already contained in it as we like. Similarly, the appearance of a pair of contradictory elements is a condition for the vanishing of the existential, if it be not negated; otherwise a condition for its becoming unity.

We approach now the consideration of a matter of the first importance, involving as it does, the creation of an infinite series of meanings of the "true" and the "false," together with an indication of how each one of these distinctions can be uniquely defined. We assume as true, for all meanings of the terms and for all meanings of $p$,

$$
p<(p)
$$

and we assert, moreover, that the converse,

$$
(p)<p,
$$

is true for not all meanings of the terms.

Now by allowing $p$ to take on the values $p^{\prime},(p),(p)^{\prime}$, etc. and by the contradiction and interchange of antecedents and consequents, we should obtain a series of results that express the relation of the existentials of first and second order and beyond to one another and to the free variable within the propositional universe. These results are represented diagrammatically in the following table, the longer space being understood to "contain" the smaller spaces which it extends beyond.

$\frac{\frac{((p)) \mid((p))^{\prime}}{(p) \mid(p)^{\prime}}}{\frac{\left((p)^{\prime}\right)^{\prime} \mid\left((p)^{\prime}\right)}{\left(p \mid p^{\prime}\right.}}$


We are now in a position to define each one of these meanings of "true" and "false." If we write

$$
\begin{aligned}
& f(p)=p \text { is false, } \\
& t(p)=p \text { is true, }
\end{aligned}
$$

it will be clear that $t=f^{\prime}$, no matter what value $f$ may have, and that, consequently, $t$ will be unambiguous as soon as $f$ has been defined.

Suppose, for example, we wish $f(p)$ to mean $p^{\prime}$. It will be enough to require $f$ to satisfy the equation

$$
f(f)=f^{\prime},
$$

for while there is an infinite series of meanings of "false" that satisfy the implication

for example,

$$
f(f)<f^{\prime}
$$

$$
(p)^{\prime}, \quad((p))^{\prime}, \quad\left((p)^{\prime}\right), \cdots,
$$

and which do not satisfy the converse, and while there is an infinite series of distinct meanings of "false" that satisfy the implication

for example,

$$
f^{\prime}<f(f)
$$

$$
\left(p^{\prime}\right), \quad\left(\left(p^{\prime}\right)\right), \quad\left(\left(p^{\prime}\right)^{\prime}\right)^{\prime}, \cdots,
$$

and which do not satisfy the converse, there is only one meaning of "false," given by

$$
f(p)=p^{\prime}
$$

which satisfies both implications at once. Accordingly $f=p^{\prime}$ is unique and $t=p$.

Any meaning of "false" from among the members of the infinite series of meanings that this term can have, can be uniquely determined by requiring $f$ to satisfy a given equation whose form can be found at once by inspection. Thus, if

$$
f(p)=p<o, \quad t(p)=(p<o)^{\prime},
$$


is the meaning of "true" and "false" desired, we assume $f$ and $t$ to satisfy respectively the equations

$$
f(f)=f<o, \quad t(t)=(t<o)^{\prime},
$$

for these solutions will prove to be unique. If

$$
f(p)=\left(p^{\prime}<o\right)^{\prime}, \quad t(p)=p^{\prime}<o,
$$

are the meanings we choose, we require $f$ and $t$ to satisfy, respectively,

$$
f(f)=\left(f^{\prime}<o\right)^{\prime}, \quad t(t)=t^{\prime}<0,
$$

and so on.

The unique solution of the equation $f(p)=0$ is $p=i$ for all meanings of $f$, as will appear as the result of a complete induction,

$$
f(p)=p^{\prime}, \quad(p)^{\prime}, \quad\left(p^{\prime}\right), \quad \text { etc. }
$$

Hence $p$ is true absolutely, that is, for all meanings of $t$,

$$
t(p)=p, \quad(p), \quad\left(p^{\prime}\right)^{\prime}, \quad \text { etc. },
$$

if $f$ satisfy the implication

$$
f<f(f) \text {. }
$$

The unique solution of the equation $f(p)=i$ is $p=o$ for all meanings of $f$. Hence $p$ is false absolutely, if $f$ satisfy the implication*

$$
f(f)<f
$$

An interesting application of our theory will result if we should now go on to specialize the meaning of $p$ and under-

* For further results that concern the theory of multiple implication see the writer's Symbolic Logic, (N. Y., Crofts, 1927). For a brief history of the problem of Epimenides as of other insolubilia see Professor E. R. Guthrie's admirable little monograph entitled The Paradoxes of $M r$. Russell. This work, little known because privately printed, has not attracted the attention it deserves. 
stand $p$ to mean "this proposition." We should then have or, if we like,

$$
f(p)=\text { this proposition is false; }
$$

$$
\text { 1. } f(1) \text {. }
$$

It is this situation that is supposed to give rise to a paradox, for it is supposed to lead to the identity of $f$ with its own contradictory. Thus if 1 . is $o, f(1)$ is $i$, if 1 . is $i, f(1)$ is $o$ and 1. and $f(1)$ are taken to be the same thing. The difficulty here depends on the assumption that every meaningful assertion must be specifically either zero or one, that is, must be a proposition, within Russell's understanding of that term. The solution to the difficulty will appear as soon as this limitation has been removed. Let us begin by defining the parts that enter into our assertion. The meaning of "false" has been defined already. It remains to define "this proposition."

We have here, perhaps, a range of choice in definition but let us require $p$ to satisfy the equation

$$
\{f<f(f)\}+\{f(f)<f\}=i .
$$

If this condition be understood to stand for the meaning of $p$, it will be seen to have two unique solutions, $p=0$ and $p=i$. This, then, is the solution of the difficulty involved in the assertion

$$
\text { "this proposition is false," }
$$

or. as we might indicate it,

$$
\text { 1. } f(1) \text {. }
$$

That is, if 1 . is two-valued, being ambiguously zero or one, then $f(1)$ is similarly two-valued and no contradiction can arise.*

*We must not, of course, on this account assume the "identity" of $p$ and $f(p)$. To do so would be to fall into the familiar fallacy of confusing the use of "all" in the collective and distributive sense. For all meanings (collectively) $p$ and $f(p)$ are the same, but they are not equal for all meanings, where "all" has the meaning of "every." 
The same solution can be shown to hold in the more general case. Consider a "cycle" of three assertions of the form

$$
\text { 1. } f(2) \quad 2 . f(3) \quad 3 . f(1)
$$

We may now attach to these too a determinate and consistent meaning. From the symmetry of the situation we may take it for granted that each one has the same general character and that this general character is the one that we assumed above. That is, these three assertions are to be conceived as two-valued in the sense defined. Since 1, 2 and 3 , then, are two-valued, so will the following be twovalued in the same sense, viz.

$$
f(1) \quad f(2) \quad f(3)
$$

and for two reasons. In the first place, because if $p$ is twovalued so is $f(p)$, (for the condition which $p$ is required to satisfy, may be regarded equally as the condition which $f(p)$ is required to satisfy, for all meanings of $f$, and there are two and only two such values, viz., $f(p)=o$ and $f(p)=i$ ), and in the second place, because they are repetitions of 1,2 and 3 . Consequently, no contradiction can arise from the simultaneous assertion of 1,2 and 3 . The same remarks will evidently hold of a "cycle" of any number of assertions of the same form and the generality of the method is evident.

The University of Pennsylvania 